\title{
RF Surface Resistance Study of Non-Evaporable Getter Coatings
}

\author{
Oleg B. Malyshev ${ }^{1,2, *}$, Lewis Gurran ${ }^{1,2,3}$, Philippe Goudket ${ }^{1,2}$, Kiril Marinov ${ }^{1,2}$, \\ Stuart Wilde ${ }^{1,4}$, Reza Valizadeh ${ }^{1,2}$ and Graeme Burt ${ }^{2,3}$ \\ ${ }^{1}$ ASTeC, STFC Daresbury Laboratory, Daresbury, Warrington, WA4 4AD Cheshire, UK \\ *e-mail address: oleg.malyshev@stfc.ac.uk \\ ${ }^{2}$ Cockcroft Institute, Daresbury, Warrington, WA4 4AD Cheshire, UK \\ ${ }^{3}$ Engineering, Lancaster University, Cockcroft Institute, Lancaster, UK \\ ${ }^{4}$ Loughborough University, Loughborough, UK
}

\begin{abstract}
In many particle accelerators the beam parameters could be affected by the beam pipe wakefield impedance. It is vital to understand how the wakefield impedance might vary due to various coatings on the surface of the vacuum chamber, and this can be derived from surface resistance measurements. The bulk conductivity of two types of NEG films (dense and columnar) is determined. This is achieved by measuring the surface resistance of NEG-coated samples using an RF test cavity and fitting the experimental data to a standard theoretical model. The conductivity values obtained are then used to compare resistive wall wakefield effects in beam pipes coated with either of the two types of film.
\end{abstract}

Keywords: surface resistance, wakefield impedance, NEG coating, PVD

PACS: $07.30 . \mathrm{Kf}, 29.20 .-\mathrm{c}, 29.27 . \mathrm{Bd}, 81.15 . \mathrm{Cd}, 81.70 . \mathrm{Ex}, 83.60 . \mathrm{Np}$ 


\section{INTRODUCTION}

A beam vacuum chamber interacts with the beam in many ways: among these is wakefield generation. In general, the wakefield impedance of a chamber depends on the material used, its surface characteristics and the chamber geometry $[1,2]$

A Non-Evaporable Getter (NEG) coating invented in the 1990s at CERN [3,4] provides the best vacuum solution for many accelerators such as LHC [5], Soleil [6] and MAX-IV [7] or key components in others such as undulator vacuum chambers in ESRF [8], DLS [9], ELETTRA [10] and many others. However, its impact upon wakefield generation in the beam vacuum chamber needs to be understood.

The aims of this work were:

- Verification of the surface resistance $\left(R_{S}\right)$ measurement method with known materials such as $\mathrm{Cu}, \mathrm{Al}$ and $\mathrm{Nb}$.

- A comparison of the surface resistance of a series of NEG films with columnar or dense structures, deposited on $\mathrm{Cu}$ or $\mathrm{Si}$ substrates at a range of thicknesses.

- Then, if possible, calculating bulk conductivity values for these two types of coatings.

- Finally, using these results to calculate the wakefield impedance. This could later be used to determine the impact of the wakefield effects on a beam.

\section{Method}

In this work, a RF cavity where the sample constitutes one of the walls is used to measure the surface resistance of the electrically conductive samples via measurement of the cavity quality factor $Q_{0}$.

The cavity geometry developed for these measurements, shown in Fig. 1, consists of two parts which are the body of the cavity and a planar sample, separated by an air gap. This presents the advantage of not requiring any contact between the sample and the cavity which makes changing the sample much simpler and removes an uncertainty with respect to the RF contact between two parts. However, in order to keep the RF power within the cavity the cavity body has a number of RF chokes. 


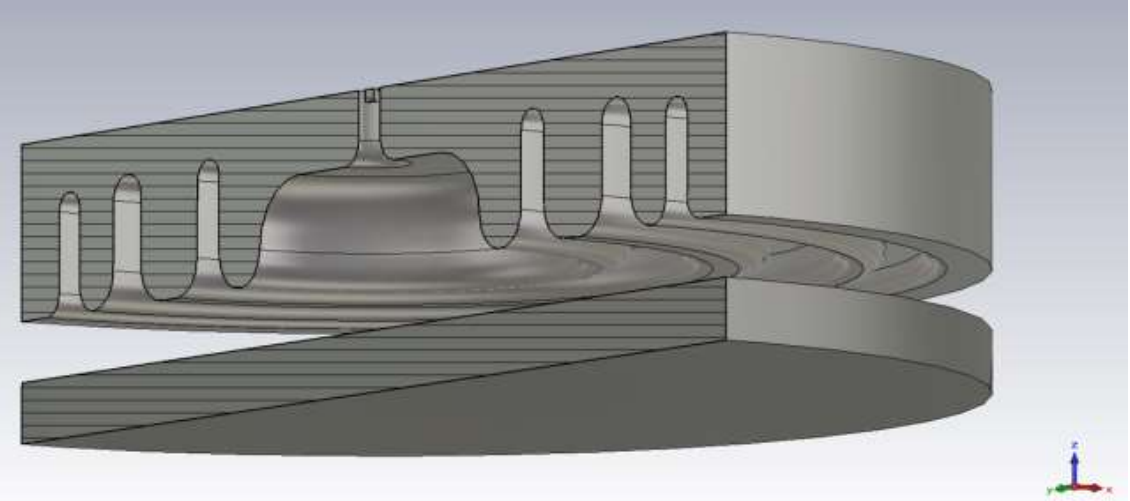

FIG. 1. A schematic of the triple choke RF cavity above a sample.

The field pattern in the cavity has been modelled via electromagnetic simulations using CST Microwave Studio [11]. The use of a double-choked pillbox-type cavity, for surface resistance measurements was described in [12]. A new three-choked cavity designed for improved leakage mitigation was used for this work and can be seen in Fig. 2. The cavity was manufactured from aluminium by Niowave Inc. (NIOWAVE Inc., 1012 N Walnut St, Lansing, MI 48906). This 7.8 GHz cavity allows the testing of 100-mm diameter samples separated from the cavity body by 1$3 \mathrm{~mm}$.

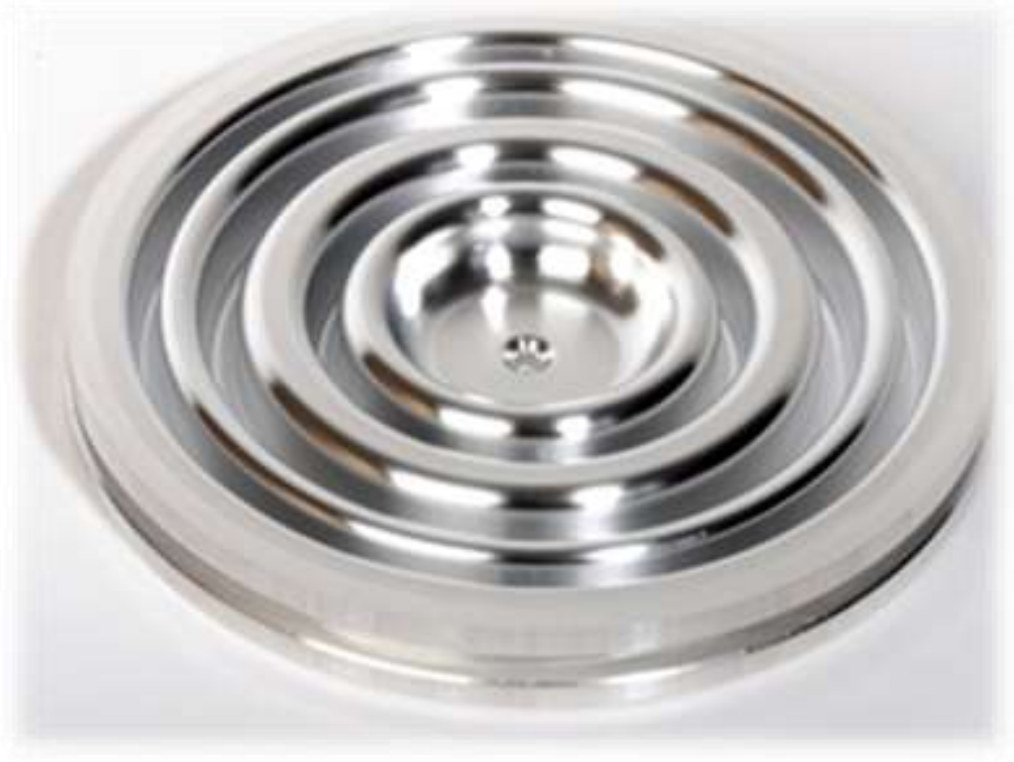

FIG. 2. The three-choked $7.8 \mathrm{GHz} \mathrm{Al}$ test cavity. 


\subsection{Calculation of surface resistance from first principles}

When an alternating magnetic field is applied to a conducting surface a surface current is induced which falls off exponentially over the characteristic 'skin depth' of the conducting material [13]. This current will absorb a time-averaged power from the AC field as

$$
P=\frac{1}{2} \iint R_{S}|\mathbf{H}|^{2} d S ;
$$

where $\boldsymbol{H}$ is the applied magnetic field strength and $R_{S}$ is the surface resistance. For a perfectly smooth, normally conducting material $R_{S}$ can be given as

$$
R_{S}=\sqrt{\frac{\pi f \mu_{0}}{\sigma}} ;
$$

where $f$ is the $\mathrm{AC}$ frequency and $\sigma$ is the bulk conductivity of the conductor. Eq. (2) is derived with the assumption that the displacement current term in Ampere's law is negligible compared to the conduction current term. This is valid at relatively low frequencies and in good conductors. The leading-order relative error associated with Eq. (2) is $\omega \varepsilon_{0} /(2 \sigma)$, where $\omega=2 \pi f$ and $\varepsilon_{0}$ is the vacuum permittivity. Thus, this error is negligible in copper at $7.8 \mathrm{GHz}$, but in a lower conductivity material e.g. $\sigma \sim 10^{4} \mathrm{~S} / \mathrm{m}$ and at higher frequencies $f \sim 10 \mathrm{THz}$ the relative deviation is $\omega \varepsilon_{0} /(2 \sigma) \sim 3 \%$. For a RF cavity the unloaded quality factor, $Q_{0}$, is defined as follows:

$$
Q_{0}=\frac{\omega_{0} U}{P} ;
$$

where $U$ is the stored energy in the cavity volume and $\omega_{0}=2 \pi f_{0}$ is the resonant frequency of the cavity. This $Q$-factor is independent of field amplitude. Where radiative losses from the chokes are small, it can be easily measured experimentally with a method described later. The timeaveraged stored energy can also be calculated from the magnetic field strength as

$$
U=\frac{\mu_{0}}{2} \iiint|\mathbf{H}|^{2} d V .
$$

The material-dependent part of $Q_{0}$ and $R_{S}$ can be separated from the field-dependent part by defining a geometry factor, $G$ :

$$
G=\frac{\omega_{0} \mu_{0} \iiint|\mathbf{H}|^{2} d V}{\iint|\mathbf{H}|^{2} d S}
$$


Since the cavity is made from two separate parts (the choked test cavity and the planar sample) which could be made of different materials the power loss can be split into two terms, one for each part:

$$
P=\frac{1}{2} \iint_{\text {sample }} R_{S}^{\text {sam }}|\mathbf{H}|^{2} d S+\frac{1}{2} \iint_{\text {cavity }} R_{S}^{\text {cav }}|\mathbf{H}|^{2} d S
$$

Assuming that the sample and test cavity each have a uniform surface resistance, $R_{S}^{\text {sam }}$ and $R_{S}{ }^{c a v}$ respectively, this allows the $Q_{0}$ factor to be expressed as

$$
Q_{0}=\frac{\omega_{0} \mu_{0} \iiint_{S}|\mathbf{H}|^{2} d V}{R_{S}^{\text {sam }} \iint_{\text {sample }}|\mathbf{H}|^{2} d S+R_{S}^{\text {cav }} \iint_{\text {cavity }}|\mathbf{H}|^{2} d S} .
$$

The 'sample ratio', $p_{s}$, and the 'cavity ratio' $p_{c}$, are introduced to simplify the equations. They are the fraction of the total integral of $|\boldsymbol{H}|^{2}$ that is integrated over the sample and test cavity surfaces, respectively:

$$
p_{s}=\frac{\iint_{\text {sample }}|\mathbf{H}|^{2} d S}{\iint_{\text {sample }}|\mathbf{H}|^{2} d S+\iint_{\text {cavity }}|\mathbf{H}|^{2} d S} ; \quad p_{c}=1-p_{s} .
$$

Eq. (7) can therefore be simplified and rearranged for $R_{S}{ }^{\text {sam }}$ as:

$$
R_{S}^{\text {sam }}=\frac{G Q_{0}^{-1}-R_{S}^{c a v} p_{c}}{p_{s}}
$$

The magnetic field distribution in the cavity was calculated using CST Microwave Studio. For our cavity, $G$ is calculated to be $235 \Omega$. Additionally, the software calculates the field ratios $p_{s}$ and $p_{c}$, for a case using perfect electric conductor (PEC) boundary conditions, to be $p_{c}=0.625$ and $p_{s}=0.375$.

Hence, for known test cavity surface resistances $R_{S}{ }^{c a v}$ (from the study carried out in [12]) and measured $Q_{0}$, the surface resistance of the sample can be calculated.

\subsection{Calculation of surface resistance from first principles}

The $Q_{0}$ represents the Ohmic heating in the cavity walls. However, the cavity requires an RF coupler in order to feed power in and out of the cavity. The coupler will also have its own quality factor, $Q_{E}$, defined as 


$$
Q_{E}=\frac{\omega_{0} U}{P_{E}}
$$

where $P_{E}$ is the power that travels back up the coupler when connected to a perfectly matched load. Any RF leakage through the chokes would add an additional non-Ohmic loss to the cavity which would appear as a radiation resistance in parallel with the cavity shunt impedance, and has an associated quality factor, $Q_{G}$. This gives the cavity and coupler a combined, or loaded, quality factor, $Q_{L}$ of

$$
\frac{1}{Q_{L}}=\frac{1}{Q_{0}}+\frac{1}{Q_{E}}+\frac{1}{Q_{G}} .
$$

The loaded quality factor is inversely proportional to the half-power bandwidth $\Delta f$ of the cavity resonance such that

$$
Q_{L}=\frac{f_{0}}{\Delta f} .
$$

Hence, the loaded $Q$-factor can easily be measured with a Vector Network Analyser (VNA, here an Agilent E5072) by measuring the resonant frequency (where the reflected power is at a minimum) and half-power points (where, with the cavity in the detuned open position, the imaginary component of the reflected voltage ratio $S_{11}$ is at a local maximum or minimum.

In order to measure the Ohmic $Q_{0}$ we must consider the minimum reflected power. With a forward power travelling into the cavity, initially when the cavity is empty almost all power is reflected back along the input cable from the coupler-cavity boundary. However, as the cavity field starts to build up, some of the power in the cavity is coupled to the input coupler creating a backwards wave which is $180^{\circ}$ out of phase with the reflected wave. This cancels out some of the reflected wave, hence the coupling parameter $\beta$ is defined as

$$
\beta=\frac{Q_{E}^{-1}}{Q_{L}^{-1}-Q_{E}^{-1}}=\frac{Q_{E}^{-1}}{Q_{0}^{-1}+Q_{G}^{-1}} ;
$$

and can be measured using the reflection coefficient $S_{11}$ : the minimum value, $S_{11}^{\min }$, at the resonant frequency and the maximum value, $S_{11}^{\max }$, just off band. When the probe is overcoupled, as for our measurements,

$$
\beta=\frac{S_{11}^{\max }+S_{11}^{\min }}{S_{11}^{\max }-S_{11}^{\min }}
$$


Therefore Eq. (11) can be rewritten as

$$
Q_{L}^{-1}=(\beta+1)\left(Q_{0}^{-1}+Q_{G}^{-1}\right) .
$$

$Q_{G}$ and $Q_{0}$ cannot be separated by measurement of $Q_{L}-$ in the equivalent circuit model of the cavity, they both contribute to the resistance. $Q_{0}$ can only be calculated with eq. (15) when $Q_{G}>>$ $Q_{0}$ or if $Q_{\mathrm{G}}$ is defined by other means as described in Section 3.1.

\subsection{Calculation of cavity surface resistance}

The quality factor $Q_{0}$ can be determined for any combination of cavity and sample materials using Eq. (15). This can then be inserted into Eq. (9) where the sample surface resistance is a function of unknown cavity surface resistance. While the conductivity of pure metals is normally well known, the surface resistance is also affected by surface contaminants, impurities and surface roughness. As a result, the surface resistance of the cavity and a sample plate of the same material can be different. Therefore for $N$ studied samples there are $N+1$ unknown values of surface resistance in a system of $N$ equations; this system of equations can't be solved. However, by using a second cavity with a different $R_{S}$ one can have $2 N$ equations for $N+2$ unknown surface resistances; this system of equations can have a solution. In these measurements two identical cavities were used, one made of aluminium and the other of niobium. Different samples, made of aluminium, niobium and 304L stainless steel (SS) as well as three copper samples (bulk plate and two PVD-deposited films to give a range of surface roughness) were used. This gave us 12 different combinations and hence 12 independent $Q_{0}$ measurements. The $12 Q_{0}$ measurements were inserted into Eq. (9) giving a system of 12 linear equations with eight unknowns, the surface resistances of each cavity and sample plate.

In practice, measurement errors in $Q_{0}$ mean that no single solution satisfies every equation. A best fit was then made to the data giving values for each of the surface resistances, including that of the cavity. The measurements were also repeated multiple times to improve the confidence in each individual $Q_{0}$ measurement. 


\subsection{Analytical models}

In this section, the equations modelling the surface resistance of the structures under investigation are discussed. The expressions for the surface impedance of a planar metallic film deposited on a substrate (dielectric or metallic) are straightforward to derive by following the standard approach employed in calculating the transmission and reflection coefficients in layered media [14]. These equations are also known from transmission line theory [15]. An alternative derivation relying on equivalent circuits can be found in [16].

The analysis begins by considering a NEG film of thickness $d_{1}$ deposited on a copper substrate much thicker than the skin depth in copper at the operating frequency $f=\omega / 2 \pi=7.8$ GHz. A layer of lossless magneto-dielectric of thickness $d_{2}$ (e.g. an oxide layer) is sandwiched between the NEG film and the copper substrate. By applying transmission line theory it can be shown that the surface impedance of this multi-layer structure is given by

$$
Z_{S}=Z_{1} \frac{\left(Z_{1}+Z_{23}\right)-\left(Z_{1}-Z_{23}\right) \exp \left(2 i k_{1} d_{1}\right)}{\left(Z_{1}+Z_{23}\right)+\left(Z_{1}-Z_{23}\right) \exp \left(2 i k_{1} d_{1}\right)}
$$

where the NEG film is described by its characteristic surface impedance $Z_{1}=\sqrt{\omega \mu_{0} / 2 \sigma_{1}}(1-i)$, wavenumber $k_{1}=\sqrt{\omega \mu_{0} \sigma_{1} / 2}(1+i)$ and bulk conductivity $\sigma_{1}$. The quantity $Z_{23}$ is the effective surface impedance of the structure underneath the NEG layer, consisting of the magnetodielectric layer of thickness $d_{2}$ on top of the copper substrate. $Z_{23}$ is given by

$$
Z_{23}=Z_{2} \frac{\left(Z_{2}+Z_{3}\right)-\left(Z_{2}-Z_{3}\right) \exp \left(2 i k_{2} d_{2}\right)}{\left(Z_{2}+Z_{3}\right)+\left(Z_{2}-Z_{3}\right) \exp \left(2 i k_{2} d_{2}\right)}
$$

where $Z_{2}=\sqrt{\mu_{0} \mu(\omega) /\left(\varepsilon_{0} \varepsilon(\omega)\right)}$ is the wave impedance of the oxide layer, $\varepsilon(\omega)$ and $\mu(\omega)$ are its permittivity and permeability, respectively, at the operating frequency, $k_{2}=\sqrt{\varepsilon \mu} \omega / c$ is the oxide layer wavenumber and $Z_{3}=\sqrt{\omega \mu_{0} / 2 \sigma_{3}}(1-i)$ is the characteristic surface impedance of copper $\left(\sigma_{3}\right.$ $=5.9 \times 10^{7} \mathrm{~S} / \mathrm{m}$ is the bulk conductivity of copper).

As in Eq. (2) the expressions for the wavenumber and the characteristic impedance of NEG and copper in Eqs. (16) and (17) rely on the assumption that the displacement current in Ampere's law is negligible in comparison with the conduction current in the NEG layer. Hence, the validity of Eqs. (16) and (17) is limited to the low frequency range such that $\omega \varepsilon_{0} / \sigma \square 1$. 
It can be easily shown that the following two conditions are simultaneously met: $\left|k_{2}\right| d_{2} \square 1$ and $\left|Z_{3}\right| \square Z_{2}$, or, equivalently, $\sqrt{|\varepsilon(\omega)||\mu(\omega)|} \square c / d_{2} \omega$ and $\sqrt{|\mu(\omega)| /|\varepsilon(\omega)|} \square\left|Z_{3}\right| / Z_{0}$, where $Z_{0}$ is the characteristic impedance of free space. Indeed, assuming that $d_{2} \sim 1 \mu \mathrm{m}$ the above requirements reduce to $\sqrt{|\varepsilon(\omega)||\mu(\omega)|} \square 6.1 \times 10^{3}$ and $\sqrt{|\mu(\omega)| /|\varepsilon(\omega)|} \square 8.6 \times 10^{-5}$. Therefore, Eq. (17) can be replaced with its Taylor series expansion and only the leading-order correction term is kept as follows:

$$
Z_{23} \approx Z_{3}-i \omega \mu(\omega) \mu_{0} d_{2} .
$$

It shows that the contribution of a thin, lossless magneto-dielectric layer to the surface impedance of a metallic substrate is purely reactive and it does not depend on the permittivity of the magneto-dielectric layer, but only on its permeability. However, if such a lossless magnetodielectric layer is sandwiched between two metals the surface resistance of the structure depends on the properties of the buffer layer such as thickness and magnetic permeability. The results presented in Fig. 3 have been obtained with NEG conductivity value of $\sigma_{1}=10^{6} \mathrm{~S} / \mathrm{m}$ as reported in Ref. [17]. Fig. 3 shows the surface resistance of such a sandwich structure. As can be seen, increasing either the thickness or the magnetic permeability of the buffer layer increases the surface resistance of the structure. Interestingly, if the permeability of the layer is negative $(\mu<$ 0 ) the surface resistance of the three-layer sandwich metal/buffer/metal (MBM) structure is actually lower than that of the of a metal-metal (MM) structure, in the absence of any layer. Note that lowering of the surface resistance of a MM structure by introducing a buffer layer with $\mu<$ 0 is still possible, even in the presence of appreciable losses $(|\operatorname{Re}(\mu)|=|\operatorname{Im}(\mu)|)$ in the buffer layer. The effect of losses in the buffer layer is to reduce the range of film thickness values over which a decrease in the surface resistance is observed. 


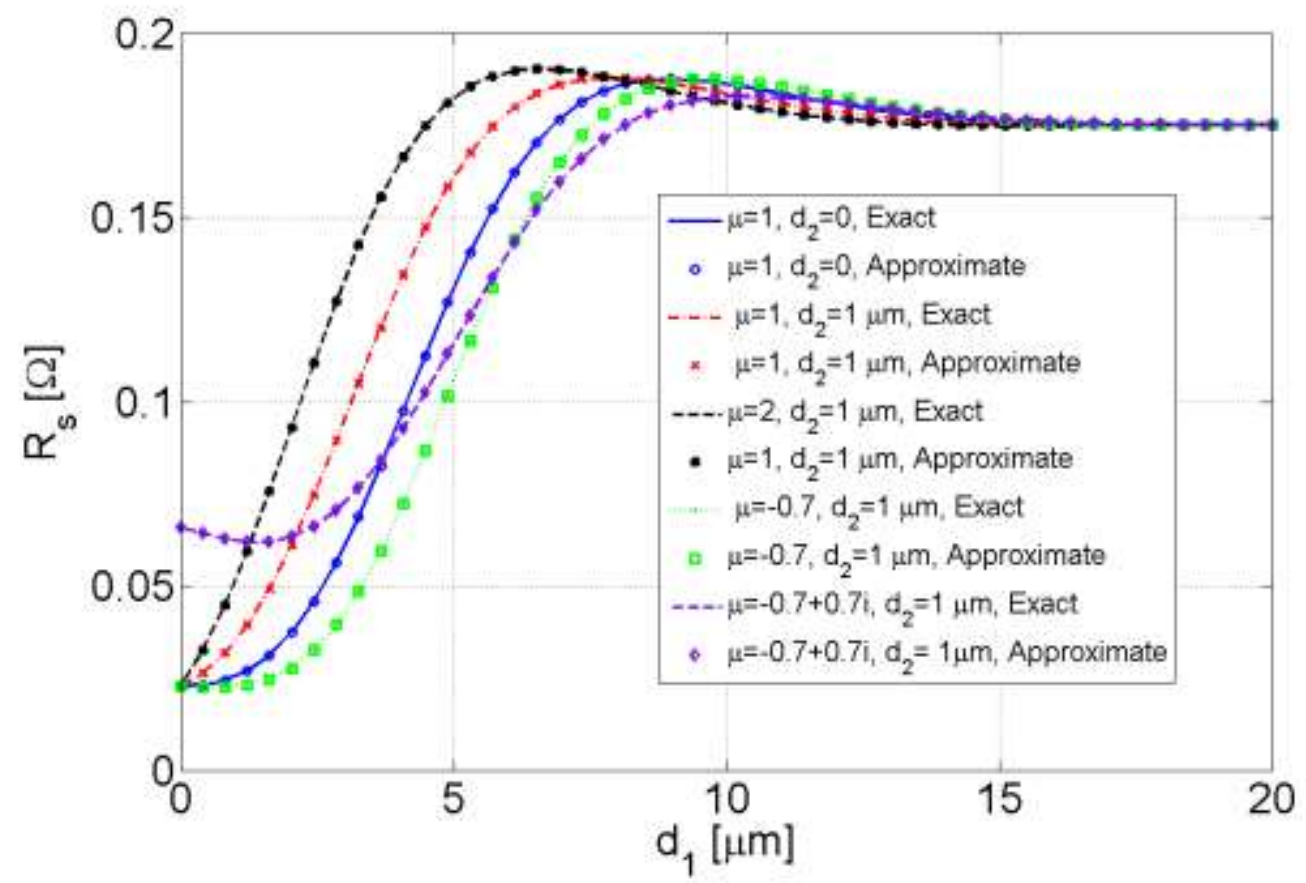

FIG. 3. Surface resistance of a NEG-oxide layer-copper structure vs. the NEG film thickness for several different permeability values of the oxide layer.

Whilst no negative permeability materials exist in nature, metamaterials research (see e.g. [18] and the references therein) has already demonstrated man-made materials exhibiting negative permeability in various frequency bands. This was first achieved at frequencies of the order of few GHz, but later studies demonstrated negative permeability in both the $\mathrm{THz}$ and the optical ranges of frequencies [19].

It should be noted that the possibility of using metamaterial coatings for resistive wakefield impedance reduction in a beam pipe has been reported earlier [20]. However, the physics behind the idea described in [20] is quite different from what has been discussed here. More details will be given elsewhere.

In the structures produced for this study the oxide layer is a few nanometres thick, hence for the purpose of the following analysis the dielectric layer can be excluded from the considerations therefore $Z_{23}=Z_{3}$. Substituting this into Eq. (16) and taking the real part yields:

$$
R_{S}=R_{1} \frac{1-\delta^{2} \exp \left(-4 \kappa_{1} d_{1}\right)-2 \delta \sin \left(2 \kappa_{1} d_{1}\right) \exp \left(-2 \kappa_{1} d_{1}\right)}{1+\delta^{2} \exp \left(-4 \kappa_{1} d_{1}\right)+2 \delta \cos \left(2 \kappa_{1} d_{1}\right) \exp \left(-2 \kappa_{1} d_{1}\right)}
$$


where $R_{S}=\operatorname{Re}\left(Z_{S}\right)$ is the surface resistance of the structure under investigation, $\kappa_{1}=\operatorname{Re}\left(k_{1}\right)$ is the inverse skin depth in the NEG film and $\delta=\left(R_{1}-R_{3}\right) /\left(R_{1}+R_{3}\right)$ is the surface resistance mismatch with $R_{1}=\operatorname{Re}\left(Z_{1}\right)$ and $R_{3}=\operatorname{Re}\left(Z_{3}\right)$.

This formalism can now be applied to a NEG film deposited on a silicon substrate, which is in turn backed-up by free space. The surface impedance of this structure is given by Eqs. (16) and (17). In the latter case $k_{2}=\sqrt{\varepsilon_{S i}} \omega / c, Z_{2}=\sqrt{\mu_{0} /\left(\varepsilon_{0} \varepsilon_{S i}\right)}$ and $d_{2}=600 \mu \mathrm{m}$ are the wavenumber, the wave impedance and the thickness of the silicon substrate, respectively; $\varepsilon_{S i}=11.65$ is the permittivity of silicon [21] and $Z_{3}=Z_{0}$. It can be shown that $Z_{2}^{2} / Z_{0} \leq\left|Z_{23}\left(d_{2}\right)\right| \leq Z_{0}$ for all values of $d_{2}$ and, hence, $\left|Z_{1}\right| \square Z_{23}$ since $\varepsilon_{S i} \square Z_{0} /\left|Z_{1}\right|$. Eq. (16) can be rewritten as follows:

$$
Z_{S}=Z_{1} \frac{Z_{1}\left(1-\exp \left(2 i k_{1} d_{1}\right)\right)+Z_{23}\left(1+\exp \left(2 i k_{1} d_{1}\right)\right)}{Z_{1}\left(1+\exp \left(2 i k_{1} d_{1}\right)\right)+Z_{23}\left(1-\exp \left(2 i k_{1} d_{1}\right)\right)} .
$$

It is easy to see that the terms proportional to $Z_{1}$ in Eq. (20) are small in magnitude compared to those proportional to $Z_{23}$, and hence, the former terms can be neglected altogether provided that the NEG layer is sufficiently thick, i.e. $d_{1} \gg d_{0}$ where $d_{0}=\left(\left|Z_{23}\right| \sigma_{1}\right)^{-1} \approx 4 \mathrm{~nm}$. The value of $d_{0}$ is calculated at $\sigma_{1}=10^{6} \mathrm{~S} / \mathrm{m}, d_{2}=600 \mu \mathrm{m}$ and $\varepsilon_{S i}=11.65$. In the latter case Eq. (20) can be simplified to

$$
Z_{S}=Z_{1} \frac{1+\exp \left(2 i k_{1} d_{1}\right)}{1-\exp \left(2 i k_{1} d_{1}\right)}
$$

Note that under these assumptions $\left(\varepsilon_{S i} \square Z_{0} /\left|Z_{1}\right|\right.$ and $\left.d_{1} \gg d_{0}\right)$ the surface impedance of the structure does not depend on the thickness and the permittivity of the dielectric substrate. Finally, taking the real part of Eq. (21) yields

$$
R_{S}=R_{1} \frac{1-\exp \left(-4 \kappa_{1} d_{1}\right)+2 \sin \left(2 \kappa_{1} d_{1}\right) \exp \left(-2 \kappa_{1} d_{1}\right)}{1+\exp \left(-4 \kappa_{1} d_{1}\right)-2 \cos \left(2 \kappa_{1} d_{1}\right) \exp \left(-2 \kappa_{1} d_{1}\right)} .
$$




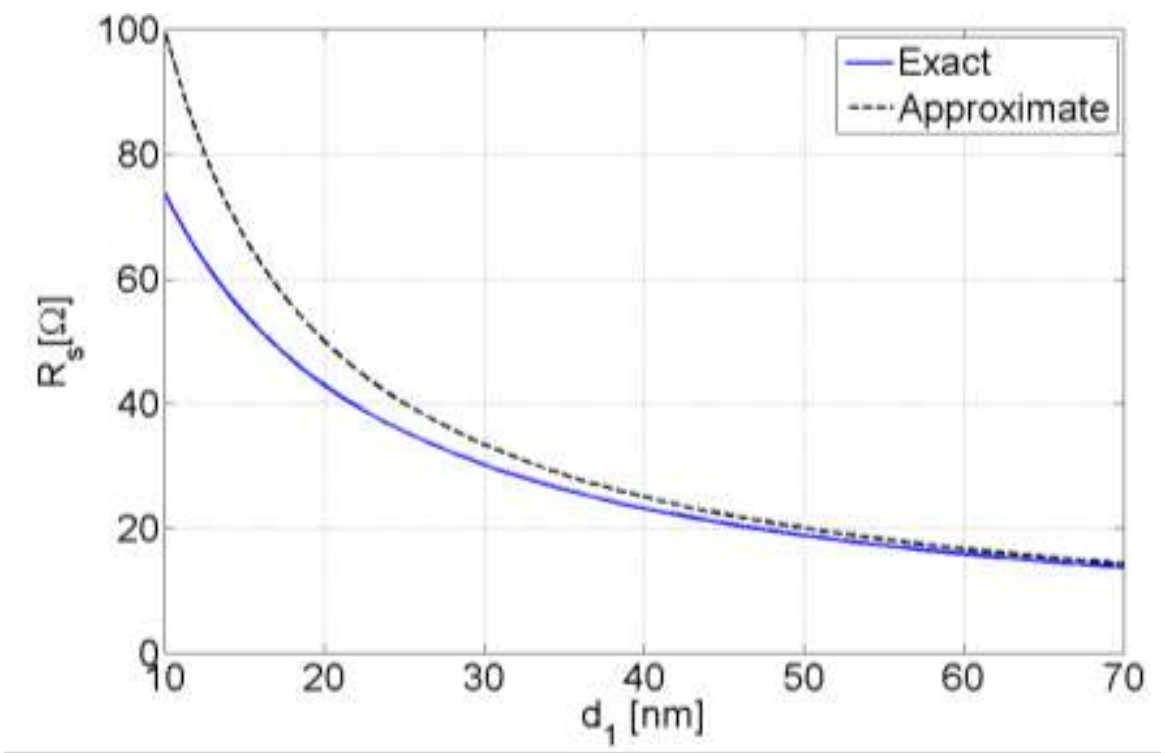

FIG. 4 Comparison between the predictions of Eq. (19) ("Exact", $\sigma_{1}=10^{6} \mathrm{~S} / \mathrm{m}, d_{2}=600 \mu \mathrm{m}, \varepsilon_{S i}=11.65$ ) and Eq. (22) ("Approximate", $\sigma_{1}=10^{6} \mathrm{~S} / \mathrm{m}$ ).

The surface resistance $R_{S}=\operatorname{Re}\left(Z_{S}\right)$ of a NEG-Si structure has been calculated using both "Exact" Eq. (19) and "Approximate" Eq. (22) (see Fig. 4). The difference between the two models becomes insignificant for NEG films of thickness $d_{1} \geq 50 \mathrm{~nm}$. In the region of NEG film thickness values relevant to our experiment the difference between the two models is less than a percent and this is considerably smaller than the experimental error.

Eqs. (19) and (22) form the basis for our subsequent analysis.

\section{MEASUREMENT RESULTS AND DISCUSSION}

\subsection{Materials used for accelerator vacuum chambers}

The materials most commonly used for accelerator vacuum chambers are copper, aluminium and stainless steel. A verification of the method described above has been performed by comparing calculated and measured values of $R_{S}$ where $R_{S}$ has been calculated from the bulk conductivity of these materials taken from the literature [22,23] and the measured surface roughness using the following formula from Ref. [24]:

$$
R_{s}=\sqrt{\frac{\mu \omega}{2 \sigma}}\left(1+\frac{2}{\pi} \arctan \left(0.7 \mu \omega \sigma R_{Q}^{2}\right)\right)
$$


where $\mu$ is the magnetic permeability, $\sigma$ is the bulk electric conductivity and $R_{Q}$ is the r.m.s. surface roughness. The results obtained with the three-choked cavity assuming $Q_{G} \gg Q_{0}$ are found to be in good agreement with calculated ones, see Table I. The values for the bulk metals agree within $11 \%$.

Table I. Calculated values of $R_{S}$ at frequency $f=7.8 \mathrm{GHz}$.

\begin{tabular}{|l|c|c|c|c|}
\hline Sample & $\begin{array}{c}\rho(\Omega \cdot \mathrm{m}) \\
\text { (from literature) }\end{array}$ & $\begin{array}{c}\text { r.m.s. } \mathrm{R}_{\mathrm{Q}}(\mathrm{m}) \\
\text { as in [12] }\end{array}$ & $\begin{array}{c}\mathrm{R}_{\mathrm{s}}(\Omega) \text { calc. } \\
\text { with Eq. }(23)\end{array}$ & $\begin{array}{c}\mathrm{R}_{\mathrm{s}}(\Omega) \\
\text { measured }\end{array}$ \\
\hline $\mathrm{Cu}$ bulk & $1.72 \times 10^{-8}[22]$ & $4.09 \times 10^{-7}$ & $2.9 \times 10^{-2}$ & $2.8 \times 10^{-2}$ \\
\hline $\mathrm{Al}$ bulk & $2.73 \times 10^{-8}[22]$ & $4.05 \times 10^{-7}$ & $3.4 \times 10^{-2}$ & $3.4 \times 10^{-2}$ \\
\hline $\mathrm{Nb}$ bulk & $1.52 \times 10^{-7}[22]$ & $\left(1.0 \times 10^{-6}\right)^{*}$ & $8.0 \times 10^{-2}$ & $7.1 \times 10^{-2}$ \\
\hline 304L SS & $7.2 \times 10^{-7}[23]$ & $1.44 \times 10^{-6}$ & $1.6 \times 10^{-1}$ & $1.7 \times 10^{-1}$ \\
\hline
\end{tabular}

* this figure is based on the manufacturer's specification of roughness rather than direct measurement.

\subsection{NEG coating on copper and silicon}

The benefit of using NEG coating on accelerator vacuum chambers is its improvement of the vacuum environment: it creates a barrier to gas diffusion from/through the vacuum chamber walls and, after activation by heating above a certain temperature for 24 hours, it provides evenly distributed pumping speed for $\mathrm{H}_{2}, \mathrm{CO}, \mathrm{CO}_{2}$ and $\mathrm{H}_{2} \mathrm{O}$ [25,26,27,28]. Different deposition parameters will vary the structure and morphology of NEG films deposited from the same 'target' (initial source of film material). Two types of Ti-Zr-Hf-V NEG film were deposited for this study: dense and columnar [29,30]. The columnar film has better pumping properties while the dense film provides a better diffusion barrier, and hence lower photon- and electron-stimulated desorption.

In this study both types of NEG film were deposited on polycrystalline copper and silicon $\mathrm{Si}(100)$ substrates. The substrate size was $100 \mathrm{~mm} \times 100 \mathrm{~mm} \times 2 \mathrm{~mm}$. Each substrate was prepared by cleaning in ultrasonic baths of acetone, then isopropanol and finally rinsed in deionised water. Deposition chamber base pressure was approximately $10^{-8} \mathrm{mbar}$. All samples were deposited using an alloy target of Ti-Zr-Hf-V with equal atomic percent at an average magnetron power of $300 \mathrm{~W}$ and using $\mathrm{Kr}$ as the sputtering gas. Each sample was set to rotate at $4 \mathrm{rpm}$ 
throughout the deposition to ensure 95\% thickness uniformity (as measured during a calibration of the system). Dense films were deposited using High Power Impulse Magnetron Sputtering (HiPIMS) with power supply settings of $2 \mathrm{kHz}$ repetition rate and a pulse width of $50 \mu \mathrm{s}$. Columnar films were deposited using a DC power supply with no substrate heating or bias. The deposition parameters are summarised in Table II.

Table II. NEG coating deposition parameters.

\begin{tabular}{|l|l|l|}
\hline Sample & Dense & Columnar \\
\hline Method & HiPIMS & DC \\
\hline Power & $300 \mathrm{~W}$ & $300 \mathrm{~W}$ \\
\hline $\mathrm{P}(\mathrm{Kr})$ & $5.3 \mathrm{mbar}$ & $40 \mathrm{mbar}$ \\
\hline Substrate temperature & $200^{\circ} \mathrm{C}$ & $\mathrm{RT}$ \\
\hline Substrate bias & $-50 \mathrm{~V}$ & $0 \mathrm{~V}$ \\
\hline
\end{tabular}

Both types of NEG film were deposited on copper and silicon substrates, and in each case with four different thicknesses, i.e. 16 samples were deposited in total. The thickness of the films was controlled by the duration of deposition, $t_{d}=1,2,6,18$ hours, and later determined accurately using a focused ion beam (FIB) cross-sectioning. High resolution planar and X-sectional views of scanning electron microscope (SEM) micrographs of dense and columnar NEG films are shown in Fig. 5. The X-sectional thickness measurement is taken at a $45^{\circ}$ tilt angle which means the scaled value should be divided by $\sqrt{2}$. The results are presented in Table III. 

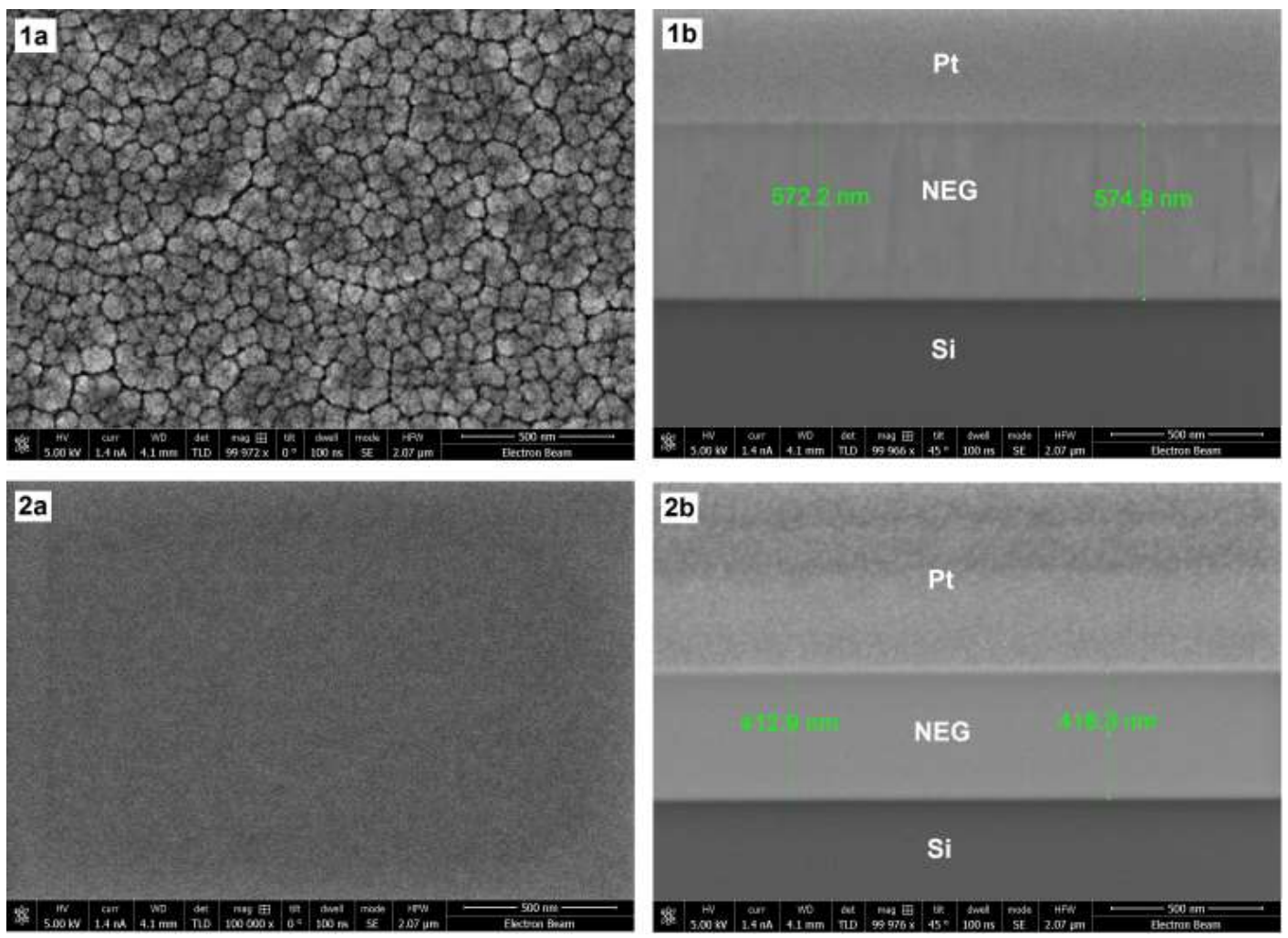

FIG. 5. The high resolution (a) planar and (b) FIB prepared X-section SEM images of columnar (1a and 1b) and dense (2a and $2 b)$ NEG films.

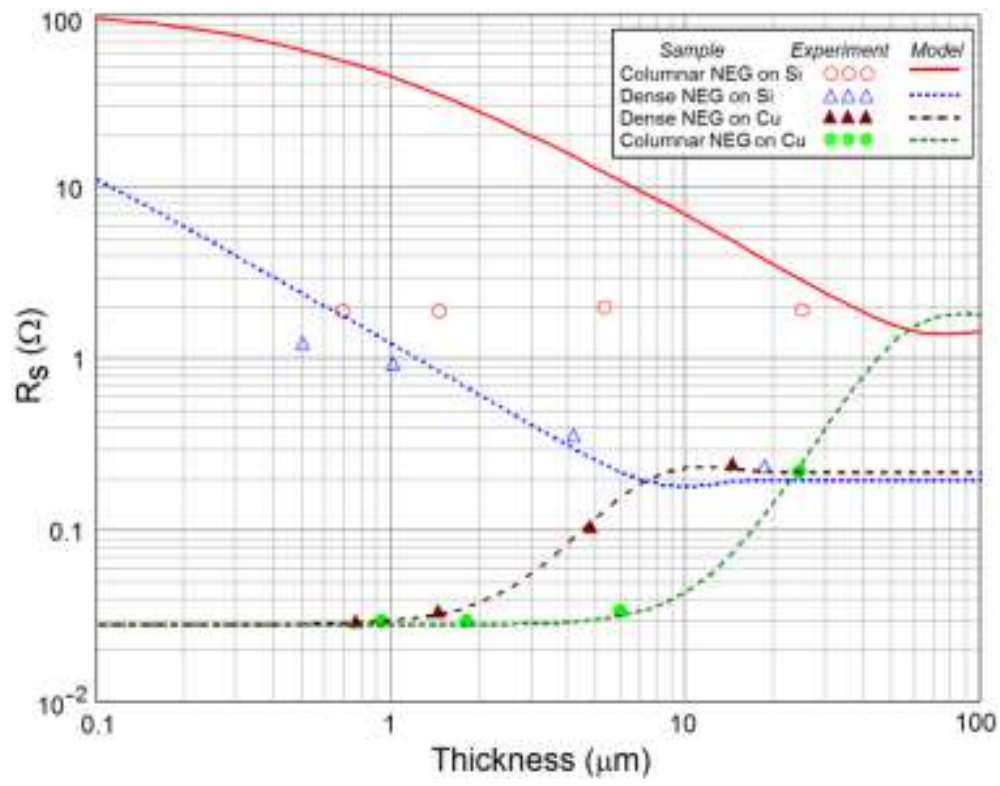

FIG. 6. The surface resistance $\mathrm{R}_{\mathrm{S}}$ of dense and columnar NEG coatings on copper and silicon substrates as a function of film thickness calculated (a) from the cavity quality factor $\mathrm{Q}_{0}$ at $7.8 \mathrm{GHz}$. and (b) the corresponding fit using the model Eqs. (19) and (22). 


\subsection{RS measurements of NEG coating on copper and silicon}

The results of $Q_{0}$ measurements at $7.8 \mathrm{GHz}$ and $R_{S}$ calculated with Eq. (9) are shown in Table III and Fig. 6 . The measurements were performed at room temperature in air using a VNA operated in single-probe mode. The measurements were all performed within a short space of time to minimise the effect of temperature and humidity fluctuations. The coupler consisted of a straight probe made of RG402 semi-rigid coaxial cable. The input probe was inserted through the central hole of the cavity (see Fig. 2). The cables were calibrated up to the SMA connectors in the probes. The input probe insertion depth was varied until the cavity was slightly overcoupled but very close to critical coupling $\left(S_{11}^{\min }<-40 \mathrm{~dB}\right)$. A set of viton spacers $(2.55-\mathrm{mm}$ thick, the same gap as simulated) was used to separate the test cavity and the sample each time.

One can see that both dense and columnar NEG coatings with thickness $d_{1}<1 \mu \mathrm{m}$ negligibly affect the copper substrates' surface resistance of $R_{S}=2.8 \times 10^{-2} \Omega$, however the surface resistance increases with larger thicknesses: for $d_{1}>1 \mu \mathrm{m}$ for dense and $d_{1}>4 \mu \mathrm{m}$ for columnar NEG film.

The uncoated silicon sample did not produce a measureable resonance with the test cavity, because the RF field was transmitted through the silicon and dissipated. The NEG films on silicon did allow measurements, albeit with caveats as discussed later. The calculated surface resistance of the thin film with thickness $d_{1}<1 \mu \mathrm{m}$ is approximately in the 1-2 $\Omega$ range. For the columnar film on Si the measured surface resistance does not measurably change with NEG film thickness over the tested range suggesting that the skin depth was significantly greater than the film thickness. For dense film however the surface resistance decreases with NEG thickness, becoming identical to NEG on copper for $d_{1}>14 \mu \mathrm{m}$ which means that this thickness of dense NEG film is sufficiently large relative to the skin depth to fully absorb the RF power. The results indicate that none of the columnar films produced for this study had a thickness greater than their skin depths.

\subsection{Measurement errors}

There are a number of possible error sources in this methodology. Based on Eq. (9) $R_{S}^{\text {sam }}$ depends on five variables with their errors: $2 \%$ for $\mathrm{G}, p_{c}$ and $p_{s}, 4 \%$ for $Q_{0}$ and $10 \%$ for $R_{S}{ }^{c a v}$, resulting in calculated $\Delta R_{S}{ }^{\text {sam }}=0.01 \Omega$ for all studied bulk metals. However, the relative error on 
$Q_{0}$ was found to be higher for thicker NEG films and the resulting $\Delta R_{S}{ }^{\text {sam }}$ is shown for each film in Table III.

Table III. $\mathbf{R}_{\mathrm{S}}$ measurements at $7.8 \mathrm{GHz}$.

\begin{tabular}{|c|c|c|c|c|c|}
\hline Sample & $\begin{array}{l}t_{d} \\
(h)\end{array}$ & $\begin{array}{l}\text { NEG thickness } \\
(\mu \mathrm{m})\end{array}$ & $\mathrm{Q}_{0}$ & $\begin{array}{l}R_{S}^{\text {sam }} \\
(\Omega)\end{array}$ & $\begin{array}{l}\Delta R_{S}^{\text {sam }} \\
(\Omega)\end{array}$ \\
\hline $\mathrm{Cu}$ & 0 & 0 & 5400 & $2.8 \times 10^{-2}$ & $8 \times 10^{-3}$ \\
\hline \multicolumn{6}{|c|}{ NEG on $\mathrm{Cu}$} \\
\hline \multirow{4}{*}{$\begin{array}{l}\text { Columnar } \\
\text { film }\end{array}$} & 1 & 0.93 & 5400 & $3.0 \times 10^{-2}$ & $8 \times 10^{-3}$ \\
\hline & 2 & 1.80 & 5400 & $3.0 \times 10^{-2}$ & $8 \times 10^{-3}$ \\
\hline & 6 & 6.03 & 5219 & $3.4 \times 10^{-2}$ & $8 \times 10^{-3}$ \\
\hline & 18 & 24.47 & 2053 & 0.22 & $1.4 \times 10^{-2}$ \\
\hline \multirow[t]{4}{*}{ Dense film } & 1 & 0.76 & 5401 & $3.0 \times 10^{-2}$ & $8 \times 10^{-3}$ \\
\hline & 2 & 1.45 & 5220 & $3.4 \times 10^{-2}$ & $8 \times 10^{-3}$ \\
\hline & 6 & 4.77 & 3284 & 0.11 & $1.0 \times 10^{-2}$ \\
\hline & 18 & 14.47 & 1899 & 0.24 & $1.6 \times 10^{-2}$ \\
\hline \multicolumn{6}{|c|}{ NEG on $\mathrm{Si}$} \\
\hline \multirow{4}{*}{$\begin{array}{l}\text { Columnar } \\
\text { film }\end{array}$} & 1 & 0.69 & 316 & 1.90 & $9 \times 10^{-2}$ \\
\hline & 2 & 1.46 & 319 & 1.88 & $9 \times 10^{-2}$ \\
\hline & 6 & 5.30 & 304 & 1.97 & $9 \times 10^{-2}$ \\
\hline & 18 & 24.92 & 313 & 1.92 & $9 \times 10^{-2}$ \\
\hline \multirow[t]{4}{*}{ Dense film } & 1 & 0.50 & 475 & 1.23 & $6 \times 10^{-2}$ \\
\hline & 2 & 1.02 & 610 & 0.94 & $5 \times 10^{-2}$ \\
\hline & 6 & 4.16 & 1419 & 0.36 & $2.1 \times 10^{-2}$ \\
\hline & 18 & 18.56 & 1939 & 0.24 & $1.5 \times 10^{-2}$ \\
\hline
\end{tabular}

The calculations performed with Eq. (9) so far assume that the losses on the sample are all Ohmic; however this may not necessarily be the case for the films deposited on silicon. If a film is not thick enough to be effectively opaque to RF (i.e. many skin depths thick) it will still carry alternating induced currents at the interface with its substrate. A sufficiently thick metallic substrate will simply dissipate all power not dissipated by the film. In the case of a thin NEG film deposited on a silicon substrate, these currents will instead radiate into the surroundings. 
Thus the $R_{S}$ calculated with Eq. (9) for a thin NEG film deposited on a silicon substrate is a lower boundary of the true value of $R_{S}$ for these samples.

An upper bound of RF leakage through the chokes QG was found by simulating the structure in CST with a tapered absorber placed around the cavity boundary to simulate a matched radiation boundary, where $Q_{G}$ was found to equal $6 \times 10^{7}$. In this simulation the cavity walls were set as having the conductivity of aluminium, however for highly conducting walls $Q_{G}$ is expected to only very weakly depend on the actual conductance value. This is likely an overestimate as alignment and manufacturing errors will decrease the choke performance hence an estimate was also made using the measurements. The lower bound can be found by combining Eqs. (9), (11) and (15) to derive a formula for $Q_{G}$ :

$$
\frac{1}{Q_{G}}=\frac{1}{Q_{L}(1+\beta)}-\frac{R_{S}^{s a m} p_{s}+R_{S}^{c a v} p_{c}}{G} .
$$

$R_{S}{ }^{\text {sam }}$ was calculated with Eq. (23) using literature data for the bulk conductivity of the metals and the r.m.s. surface roughness (see Table 1). These calculated values of $R_{S}{ }^{\text {sam }}$ were inserted into Eq. (24) along with the cavity parameters $G, p_{c}$ and $p_{s}$ calculated in CST MWS, and $R_{S}{ }^{c a v}$ calculated as in Section 2.3 to be $4.76 \times 10^{-2}$ and $9.15 \times 10^{-2} \Omega$ for the aluminium and niobium cavities, respectively. While both cavities have the same geometry in principle, the chokes' performance is very sensitive to manufacturing tolerances hence we need to calculate it for both cavities separately. Over several different combinations of materials, the r.m.s. $Q_{G}$ found was 92,000 for the aluminium cavity and 89,000 for the niobium cavity. This would introduce an additional systematic error into the measurements: up to $+5 /-0 \%$ for $\mathrm{Q}_{0}$ and up to $+0 /-12 \%$ for $R_{S}$.

Unaccounted-for possible sources of error are:

- The assumption that the metal remains in the normal skin-depth regime.

- The roughness-modified formula for $R_{S}$ is only an empirical approximation.

- The metal samples used might have been slightly different in composition and structure to those measured in the literature. Therefore, their bulk resistivity might be slightly different from that quoted.

- Surface oxidation, dirt and/or fractures beneath the surface of the samples could all also have had an effect on $R_{S}$ which is not currently quantifiable.

- The humidity and temperature of the lab was not a controlled variable. 
- Calibration to the end of the RF probe was not possible in our setup.

\subsection{Fitting the analytical model to the experimental data}

The $R_{S}{ }^{\text {sam }}$ calculated from experimental measurement has been plotted alongside the analytical predictions with use of Eqs. (19) and (22): for the four NEG coatings at various deposition thicknesses, see Fig. 6. The bulk conductivity of the NEG coatings can then be estimated from the fit. It can be noticed that good agreement has been achieved for all NEG on copper. However, the fit for dense NEG on silicon is poor for $d_{1} \leq 1 \mu \mathrm{m}$ and there is no correlation between theoretical and measured values for the columnar NEG on silicon; this can be explained by the film thickness being thinner than the skin depth. In this case the surface current flows on both sides of the NEG coating and the currents induce RF radiation in the silicon dielectric substrate, therefore Eq. (11) should be re-written as follows:

$$
\frac{1}{Q_{L}}=\frac{1}{Q_{0}}+\frac{1}{Q_{E}}+\frac{1}{Q_{G}}+\frac{1}{Q_{o l}},
$$

where $Q_{o l}$ represents other losses which are in this case the losses through the columnar NEG film and are no longer negligible. Therefore, the $R_{S}$ calculations with use of Eq. (9) for the NEG film on silicon could be invalid, at least for dense NEG with $d_{1} \leq 1 \mu \mathrm{m}$ and for the columnar NEG with $d_{1} \leq 25 \mu \mathrm{m}$. Thus the comparison of the measurements and the analytical model demonstrates that when this measurement method returns $R_{S}>1 \Omega$ the result should not be considered to be reliable.

The bulk conductivity was obtained from a best fit to the experimental data for both types of NEG coating with Eqs. (19) and (22):

- $\sigma_{d}=1.4 \times 10^{4} \mathrm{~S} / \mathrm{m}$ for the columnar NEG coating,

- $\sigma_{d}=8 \times 10^{5} \mathrm{~S} / \mathrm{m}$ for the dense NEG coating.

It is worth mentioning that the calculated value of bulk conductivity for the dense NEG coatings is exactly the same as that of bulk Ti-Zr-Hf-V alloy as measured on the 3-mm-diameter rod which was used as a target for vacuum chamber deposition in ASTeC [31].

To compare the surface resistance of dense and columnar films as a function of NEG film thickness is divided into five zones, as shown in Fig. 7:

(I) NEG coating's impact on the substrate surface resistance is negligible: $R_{S}(\mathrm{NEG}) \approx$ $R_{S}(\mathrm{Cu})$ 
(II) $R_{S}$ of dense NEG coating steadily increases to its maximum, the columnar NEG impact on $\mathrm{R}_{\mathrm{S}}$ is still negligible: $R_{S}($ dense $)>R_{S}($ columnar $) \approx R_{S}(\mathrm{Cu})$;

(III) $R_{S}$ of columnar NEG coating steadily increases and reaches a maximum value for dense NEG: $R_{S}(\mathrm{Cu})<R_{S}($ columnar $)<R_{S}($ dense $)$;

(IV) $R_{S}$ of columnar NEG coating steadily increases to its maximum: $R_{S}(\mathrm{Cu})<R_{S}($ dense $)<$ $R_{S}($ columnar);

(V) $R_{S}$ of both dense and columnar NEG do not increase further with thickness.

Due to its lower conductivity the columnar NEG coating provides negligible impact on the substrate surface resistance for larger thicknesses than the dense NEG coating; however, the latter provides the lower $R_{S}$ for thicker coating (a coating thick enough to be radioopaque). It is important to note here that the structure of NEG coating can vary with deposition conditions and parameters between the two extremes studied in this study, i.e. in general the bulk conductivity will be between measured values: $1.4 \times 10^{4} \leq \sigma_{d} \leq 8 \times 10^{5} \mathrm{~S} / \mathrm{m}$; therefore, the $R_{S}$ of such a film will start increasing in zone (II) and its maximum will be between those measured here.

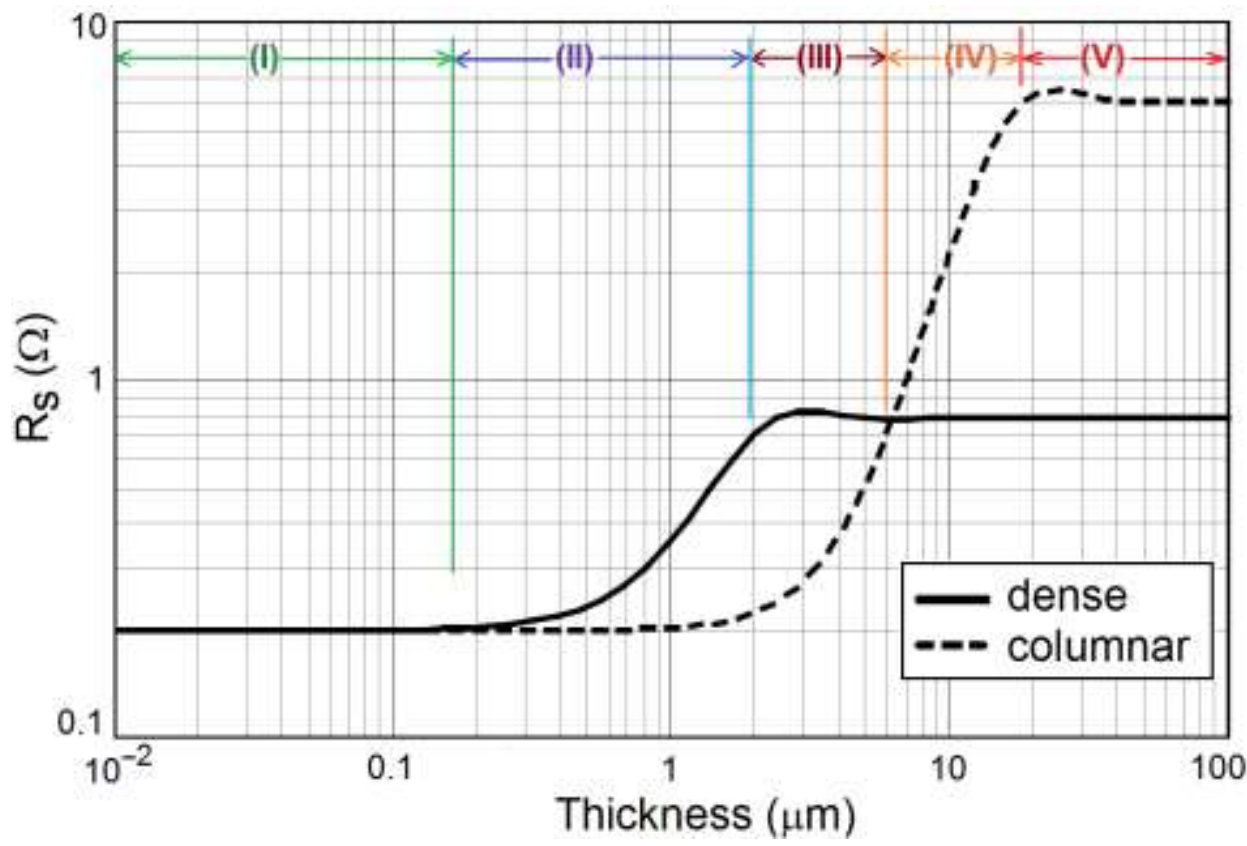

FIG. 7. A comparison of surface resistance of columnar (solid line) and dense (dashed line) NEG films, zones (I)-(V) as described in the text. 


\section{SURFACE AND WAKEFIELD IMPEDANCE}

\subsection{Resistive wall wakefield effects}

In this section the resistive wall wakefield effects in vacuum vessels coated with either dense or columnar NEG film are studied and the performance of the two vessels is compared. A Gaussian electron bunch traveling down the axis of a copper pipe of circular cross-section and radius 5 $\mathrm{mm}$ is considered. The thickness of the NEG layer coating the pipe is $d$. The standard approach $[1,2]$ to calculating the longitudinal wakefield impedance of these structures $Z_{W}(\omega)$ has been employed. It has been assumed that the bunch spectrum frequencies are within the validity of the classical free-electron conductivity model (a.k.a. Drude model) and well below the onset of interband transitions that lead to a complex dependence of conductivity on frequency (see e.g. [32]). The longitudinal wakefield impedance is in turn used to calculate the energy loss and the correlated energy spread induced in the bunch. The bunch length values range from a few femtoseconds (relevant to a modern free electron laser) up to a few picoseconds. Figure 8 shows the result. Intuitively one would expect the vessel coated with higher-conductivity material (dense NEG) to outperform that coated with the lower-conductivity material (columnar NEG). However, as Fig. 8 shows, this is not always the case. At very short bunch lengths the two types of coatings perform at comparable levels and this is related to the fact that the resistive wall wakefield generated by a very short bunch approaches a limit that does not depend on the conductivity of the vessel but only on its radius [1]. In the low-frequency regime (bunch lengths exceeding $0.5 \mathrm{ps}$ ) (Fig. 8a and $8 \mathrm{~b}$ ) the extent to which the higher-conductivity film outperforms the lower-conductivity one becomes less pronounced. Surprisingly, in this regime the energy loss caused by the lower-conductivity, columnar NEG film is lower than that caused by the higherconductivity dense film. This result can be explained by Fig. 8c. As can be seen at frequencies below $\sim 1.2 \mathrm{THz}$ the wakefield impedance of the columnar film (responsible for the energy losses) is lower than that of the dense film. For bunches of spectral width significantly less than $\sim 1.2 \mathrm{THz}$ the contribution of the sharp resonant peak displayed by the columnar NEG film is insignificant. The energy of such bunches is absorbed in both the coatings and in the copper substrate. In other words the longer bunch wakefield "sees" the high-conductivity copper and this yields a lower value of the energy loss compared to a dense film. In the latter the field is more efficiently shielded from the copper by the higher-conductivity (and lower skin depth). 

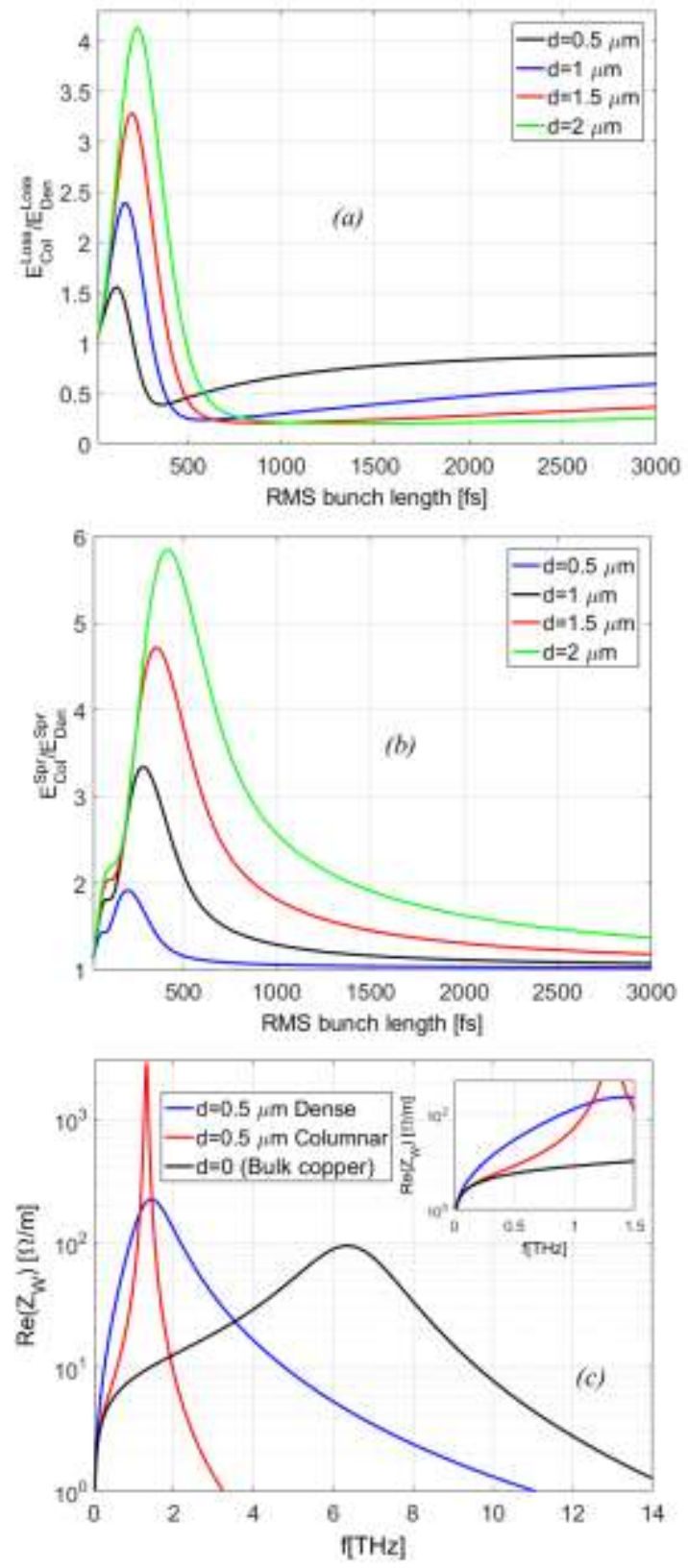

FIG. 8. Ratios between (a) the energy losses and (b) the energy spreads induced by resistive wakefields in a circular copper pipe of radius $5 \mathrm{~mm}$ coated with columnar ("Col”) and dense ("Den”) NEG layers of thickness $d$. (c) Real part of the longitudinal wakefield impedance of three copper pipes of radius $5 \mathrm{~mm}$ coated by: a layer of dense NEG ("Dense"), a layer of columnar NEG ("Columnar") $(d=5 \mu \mathrm{m})$ and an uncoated pipe ("Bulk copper"). The conductivity values for dense NEG, columnar NEG and copper are $\sigma_{\mathrm{Den}}=8 \times 10^{5} \mathrm{~S} / \mathrm{m}, \sigma_{\mathrm{Col}}=1.4 \times 10^{4} \mathrm{~S} / \mathrm{m}$ and $\sigma_{\mathrm{Cu}}=$ $5.9 \times 10^{7} \mathrm{~S} / \mathrm{m}$. It is assumed that the Drude relaxation time of both dense and columnar NEG is equal to zero and its value for copper is $\tau=27 \mathrm{fs}$. The inset shows shows the behavior of the real parts of the longitudinal impedances of the three vessels in the region below $1.5 \mathrm{THz}$. 
At frequencies below $\sim 100 \mathrm{GHz}$ the value of the impedance of the columnar film is very close to that of an uncoated copper vessel of the same radius. In this frequency range the $0.5 \mu \mathrm{m}$ columnar film is effectively transparent and the electromagnetic energy is absorbed entirely in the copper vessel.

The intermediate range of frequencies (and bunch lengths) corresponds to a situation where the bunch length is still far from the ultra-short bunch limit (where there is no dependence on the conductivity) but the bunch spectrum is sufficiently wide that the contribution of the sharp peak of the real part of the impedance of columnar NEG (see Fig. 8c) becomes dominant in the expression for the energy loss (see e.g. [2]). In this regime energy is absorbed predominantly in the coatings and the higher-conductivity material outperforms the lower-conductivity one.

\subsection{Calculation of $R_{S}$ at various frequencies}

The obtained bulk conductivity of the columnar and dense NEG coatings can be used with the analytical model for calculating the surface resistance of NEG coatings on various substrate materials and at various frequencies.

Surface resistance of NEG coatings on copper, aluminium and 304L stainless steel were calculated as a function of film thickness for dense and columnar film at the following frequencies: 1, 10 and $100 \mathrm{GHz}, 1$ and $10 \mathrm{THz}$. The results are shown in Figs. 9-11.

The frequency dependence of the results can be explained by the frequency dependence of the skin depth and the material properties and thickness of the various layers. At higher frequencies, the skin depth is shorter, and the relative effect of the first layer on the overall surface resistance is greater. Let's consider using a typical $1-\mu \mathrm{m}$ thick NEG coating. Its impact on the copper and aluminium substrate surface resistance (see Figs. 9-10) will be the following:

- negligible at $f=1-10 \mathrm{GHz}$,

- negligible with the columnar NEG but increased with the dense NEG at $f=100 \mathrm{GHz}$,

- at $f=1 \mathrm{THz}$ it will reach its maximum with the dense NEG which is higher than for the columnar NEG,

- at $f=10 \mathrm{THz}$ the surface resistance of the dense NEG is lower than that of the columnar NEG. 
For films on stainless steel the difference between the dense and columnar NEG coating is insignificant at $f=1-10^{3} \mathrm{GHz}$ and only at $f=10 \mathrm{THz}$ is the surface resistance of the columnar NEG significantly higher than of the dense NEG.

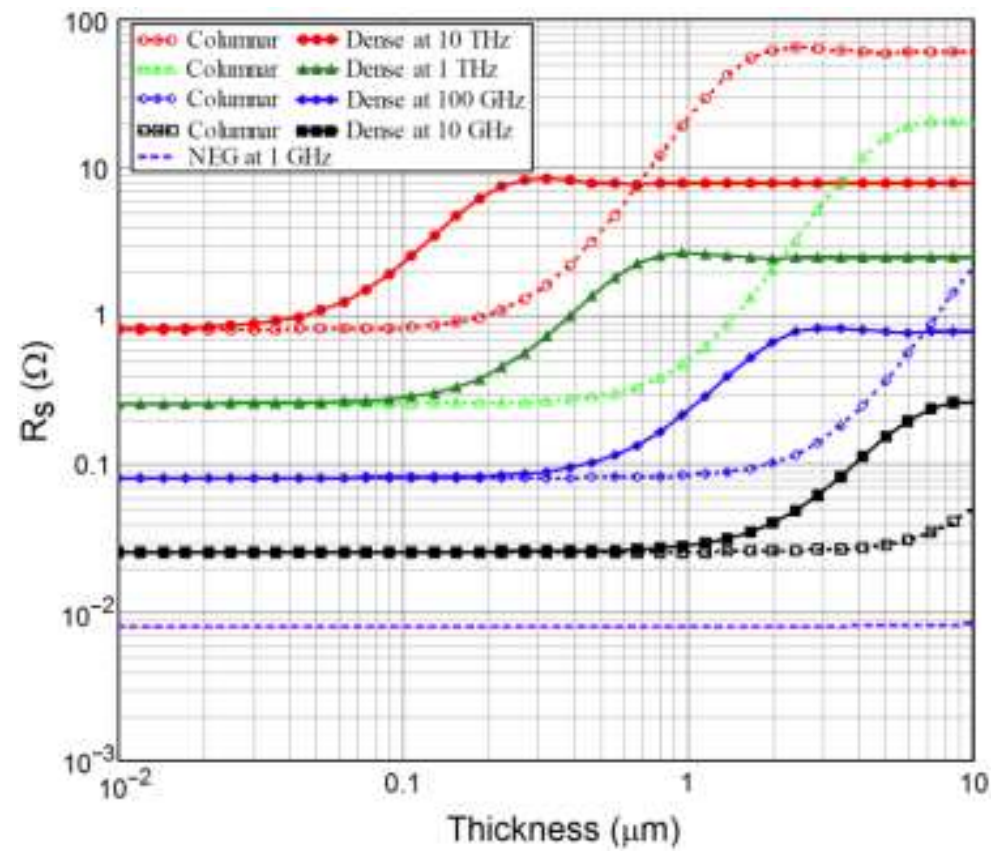

FIG. 9. The surface resistance $R_{S}$ as a function of NEG film thickness on copper at various frequencies.

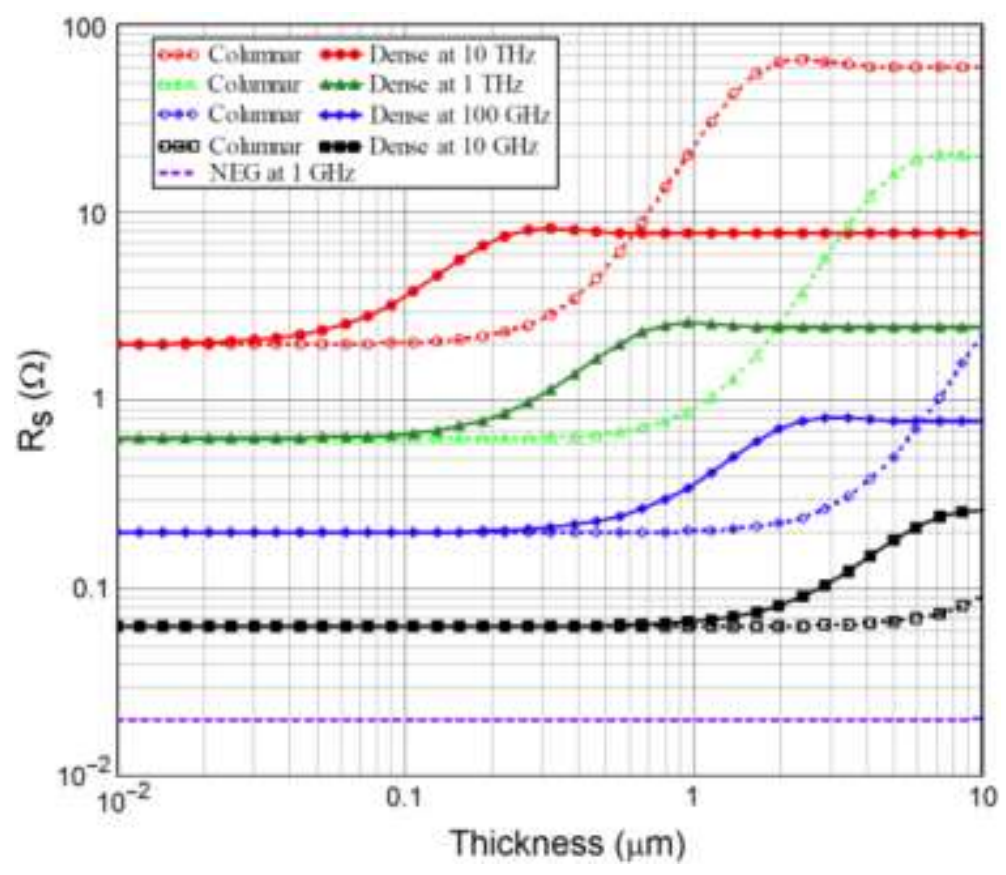

FIG. 10. The surface resistance $R_{S}$ as a function of NEG film thickness on aluminium at various frequencies. 


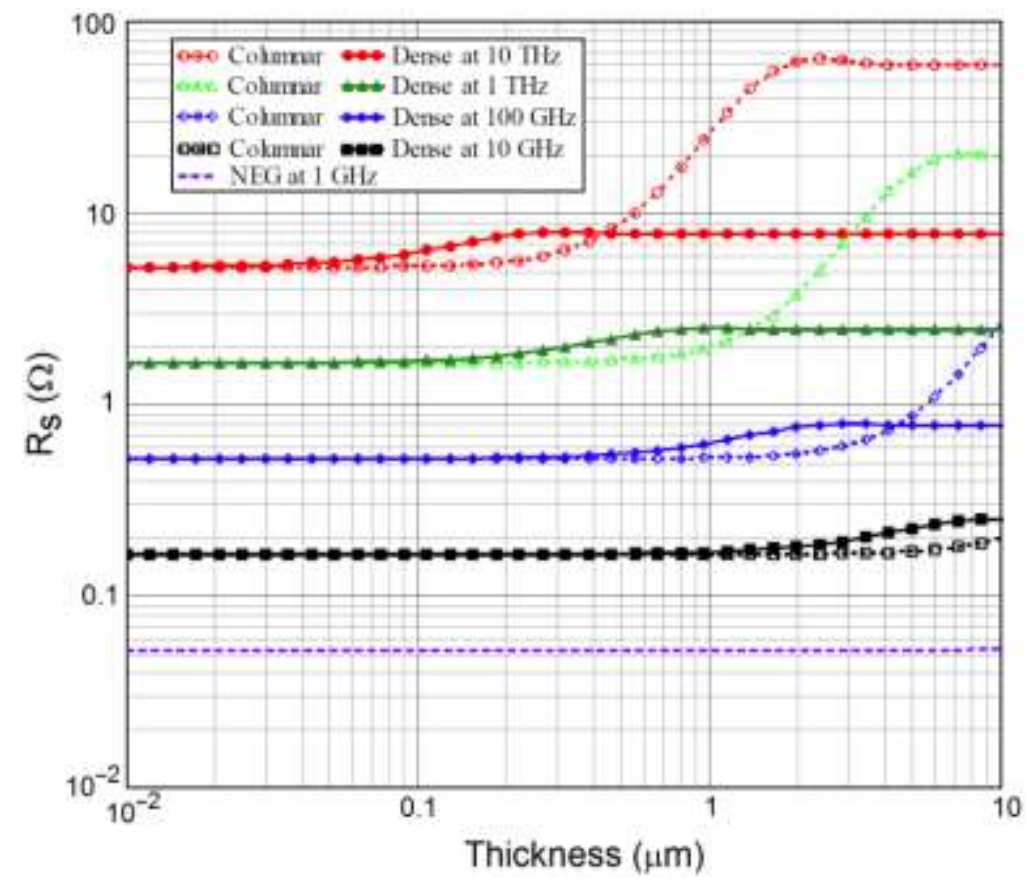

FIG. 11. The surface resistance $\mathrm{R}_{\mathrm{S}}$ as a function of NEG film thickness on 304L stainless steel at various frequencies.

\section{Conclusions}

1. The three-choked cavity is a valuable and reliable tool for measurement of the surface resistance of metal surfaces and obtaining the bulk conductance of thin films.

2. The surface resistance of two types of NEG coating (dense and columnar) was investigated at 7.8 GHz. The bulk conductivity was obtained with the analytical model: $\sigma_{d}=1.4 \times 10^{4} \mathrm{~S} / \mathrm{m}$ for the columnar NEG coating and $\sigma_{d}=8 \times 10^{5} \mathrm{~S} / \mathrm{m}$ for the dense NEG coating.

3. The standard analytical models for the surface resistance of multilayer structures and the obtained experimental results for the films deposited on copper substrates are in good agreement.

4. Based on the NEG conductivity values obtained at $7.8 \mathrm{GHz}$ (and with the assumption that the classical Drude model is still valid) the beam energy loss and the energy spread induced by resistive wall wakefield effects on the beam have been investigated and compared in vessels coated with either of the two types of film.

5. It was demonstrated that the surface resistance of copper at $\mathrm{GHz}$ frequencies increases insignificantly with a $1-\mu \mathrm{m}$ thick NEG coating. However, with the assumption that the 
classical Drude conductivity model is still valid (implying frequency-independent conductivity) the theoretical model indicates that the increase could be significant at $\mathrm{THz}$ frequencies.

\section{Acknowledgements}

This work was conducted under the aegis of the Science \& Technology Facility Council (STFC).

The authors would also like to acknowledge the support provided by ASML (Veldhoven, Netherlands).

\section{References:}

[1] B. Zotter and S. Kheifets, Impedances and Wakes in High-Energy Particle Accelerators, World Scientific (1990).

[2] A. Chao, Physics of Collective Beam Instabilities in High-Energy Accelerators, Willey\&Sons (1993).

[3] C. Benvenuti, Proc. EPAC1998, Stockholm, Sweden, p. 200 (1998).

[4] C. Benvenuti, P. Chiggiato, F. Cicoira and V. Ruzinov, Vacuum 50, 57 (1998).

[5] V. Bencini, G. Bregliozzi, V. Baglin, et al. Proc. IPAC2015, Richmond, VA, USA, p. 3100 (2015).

[6] C. Herbeaux , N. Béchu and J-M. Filhol. Proc. EPAC08, Genoa, Italy, p. 3696.

[7] S. Calatroni, P. Chiggiato, P. Costa Pinto, et al. Proc. IPAC2013, Shanghai, China, p. 3385 (2013).

[8] R. Kersevan. Proc. EPAC-2000, Vienna, Austria, p. 2291 (2000).

[9] J.D. Herbert, O.B. Malyshev, K.J. Middleman and R.J. Reid. Vacuum 73, 219 (2004).

[10] F. Mazzolini, J. Miertusova, F. Pradal, L. Rumiz. Proc. EPAC2002, Paris, France, p. 2577 (2002).

[11] CST AG, Bad Nauheimer Str. 19 Darmstadt, 64289 Germany.

[12] P. Goudket, L, Gurran, G. Burt, et al. Proc. IPAC2015, Richmond, VA, USA, p. 3235 (2015).

[13] H. Padamsee, J. Knobloch and T. Hayes, RF Superconductivity for Accelerators, Wiley, 1998.

[14] Jin Au Kong, Electromagnetic wave theory, Willey \&Sons (1990).

[15] Chien-Jang Wu, Yao-Li Chen and Tzong-Jer Yang, J Supercond Nov Magn 23, 545 (2010).

[16] http://www.gb.nrao.edu/electronics/edir/edir302.pdf.

[17] E. Koukovini-Platia, G. Rumolo and C. Zannini, Proc. IPAC, Dresden, Germany, 2384 (2014).

[18] V. Shalaev, Nature Photon. 1, 41 (2007).

[19] H. K. Yuan et al, Opt Express 15, 1076 (2007).

[20] A. Danisi, C. Zannini, A. Masi, R. Losito and B. Salvant, Proc. IPAC, Dresden, Germany, 1695 (2014).

[21] J. Krupka, J. Breeze, A. Centeno, N. Alford, T. Claussen, and L. Jensen, IEEE Transactions on Microwave Theory and Techniques 54 (2006).

[22] W. M. Haynes (ed) CRC Handbook of Chemistry and Physics, 94th Edition. CRC Press. Boca Roton, Florida, 2013; Sections 4 and 12.

[23] Stainless Steel - Grade 304 (UNS S30400). AZO Materials at http://www.azom.com/article.aspx?ArticleID=965 17/11/15.

[24] E. O. Hammerstadt and F. Bekkadal, "A Microstrip Handbook", ELAB Report STF 44 A74169, Univ. of Trondheim, Norway, 1975, pages 98-110.

[25] C. Benvenuti, A. Escudeiro Santana, and V. Ruzinov, Vacuum 60, 279 (2001).

[26] P. Chiggiato and R. Kersevan, Vacuum 60, 67 (2001).

[27] V.V. Anashin, I.R. Collins, R.V. Dostovalov, et al, Vacuum 75, 155 (2004). 
[28] O. B. Malyshev. Vacuum Systems for the ILC Damping Rings. EuroTeV report-2006-094 \& Cockcroft-06-84. [Nov 2006. http://www.eurotev.org/.

[29] O. B. Malyshev, R. Valizadeh, R. M. A. Jones and A. Hannah, Vacuum 86, 2035 (2012).

[30] O.B. Malyshev, R. Valizadeh and A. Hannah. Vacuum 100, 26 (2014).

[31] O.B. Malyshev, R. Valizadeh, B.T. Hogan and A. Hannah. J. Vac. Sci. Technol. A 32, 061601 (2014).

[32] H. Ehrenreich and H. R. Philipp, Phys. Rev. 128, 1622 (1962). 